\title{
EIGENVALUE ESTIMATES FOR STABLE MINIMAL HYPERSURFACES
}

\author{
KEOMKYO SEO
}

Abstract. In this article we provide estimates on the first eigenvalue of stable minimal hypersurfaces in a Riemannian manifold with sectional curvature bounded from below and above by negative constants. We also obtain a lower bound of the total scalar curvature of a stable minimal hypersurface if the scalar curvature of the ambient space is positive.

Mathematics subject classification (2010): 53C21, 53A10.

Keywords and phrases: Stability, minimal hypersurface, first eigenvalue, Yamabe invariant.

\section{REFERENCES}

[1] A. CANDEL, Eigenvalue estimates for minimal surfaces in hyperbolic space, Trans. Amer. Math. Soc., 359 (2007), 3567-3575.

[2] I. Chavel, Isoperimetric inequalities. Differential geometric and analytic perspectives, Cambridge Tracts in Mathematics 145, Cambridge University Press, Cambridge, 2001.

[3] J. ChoE, The isoperimetric inequality for minimal surfaces in a Riemannian manifold, J. Reine Angew. Math., 506 (1999), 205-214.

[4] P.T. Ho, Eigenvalue estimate for minimal hypersurfaces in hyperbolic space, Differential Geom. Appl., 27 (2009), 104-108.

[5] D. HofFMAn AND J. SPRUCK, Sobolev and isoperimetric inequalities for Riemannian submanifolds, Comm. Pure Appl. Math., 27 (1974), 715-727.

[6] J. Kazdan and F. Warner, Prescribing curvatures, Proc. Sympos. Pure Math., Vol. 27 (1975), 309-319.

[7] R. SCHOEN, Variational theory for the total scalar curvature functional for Riemannian metrics and related topics, Lecture Notes in Math. 1365, Springer, 1989, 120-154.

[8] R. SCHOEN AND S.-T. YAU, Existence of incompressible minimal surfaces and the topology of threedimensional manifolds with nonnegative scalar curvature, Ann. of Math. (2), 110 (1979), 127-142.

[9] R. SCHOEN AND S.-T. YAU, On the structure of manifolds with positive scalar curvature, Manuscripta Math., 28 (1979), 159-183.

[10] K. SEO, Stable minimal hypersurfaces in the hyperbolic space, to appear in J. Korean Math. Soc. 